\title{
Die Hauptprobleme der religiösen Symbolik
}

\author{
Von HARALDS BIEZAIS
}

1. Im Leben des Einzelnen als auch religiöser Gruppen ist das religiöse Symbol von zentraler Bedeutung. Damit ist auch das gesteigerte Interesse für Probleme, die mit ihm verbunden sind, zu erklären ${ }^{1}$. Ich kann hier nur auf einige dieser Probleme hinweisen, die so bedeutsam sind, daß man an ihnen nicht vorbeikommt, ohne danach seinen Standpunkt zu präzisieren. Dagegen glaube ich nicht, daß man versuchen müßte, eine mehr oder weniger vollständige systematische oder historische Übersicht über diese Probleme zu geben ${ }^{2}$, denn eine solche würde für die Erörterung der Probleme nichts Neues bringen.

1.1. Das besondere Interesse unserer Zeit am Symbol steht in engem Zusammenhang mit der Weiterbildung und Entwicklung von Ansichten, wie sie die Theoretiker der Psychoanalyse Freud und Jung geäußert haben.

Ihre Grundthese lautet, daß die Aktivitäten der menschlichen Psyche sich von Zeit zu Zeit in Symbolen äußern, welche vom Unterbewußtsein bestimmt werden ${ }^{3}$. Die symbolischen Bilder würden in Wirklichkeit durch erblicherweise übernommene Inhalte des kollektiven Unterbewußtseins bestimmt. Nur die Formen, welche diese Inhalte annehmen und durch welche sie sich äußern, würden durch die jeweiligen Erfahrungen und Erlebnisse mitbestimmt ${ }^{4}$. So wird also verständlich, daß die symbolischen Formen im Verhältnis zum Inhalt von sekundärer Bedeutung sind. Der Einfluß dieser Theorie hat sich, ungeachtet der wesentlichen biologischen Einschränkung und Einseitigkeit des Symbolbegriffs ${ }^{5}$, auch tiefgreifend auf die Forschungen über die religiöse Symbolik ausgewirkt.

\footnotetext{
1 Einen weiten Überblick über diese Forschungen bieten die entsprechenden Bibliographien und besonderen Sammlungen. Bibliographie $1968 \mathrm{ff}$; Symbolon $1960 \mathrm{ff}$. Cf. auch Giedion, 78, mit seinen Aussagen.

2 Ein Beispiel dafür, daß es nicht möglich ist, Symbole zu systematisieren und zu gruppieren, oder, richtiger, daß dies nur nach rein subjektiv ausgewählten Grundsätzen erfolgen kann, bietet Assagioli $34 \mathrm{ff}$.

${ }^{3}$ Cf. Moody $12 \mathrm{f}$.

${ }^{4} \mathrm{Ib}$.

5 Gegen Jungs Theorie hat sich Jaspers, 1946, 277, gewandt: ,In der verstehenden Psychologie ist besonders $\mathrm{zu}$ unterscheiden das Symbol als Träger persönlich gültiger Bedeutungen aus der eigenen Lebensge-
}

schichte, als Ersatzbildung usw. von dem Symbol als umgreifenden Bedeutungsträger immanenter Transzendenz. Jenes läßt Jung sich gründen im persönlichen Unbewußten, dieses im kollektiven Unbewußten." Er lehnt die Psychoanalyse und ähnliche Theorien grundsätzlich ab 1947, 1040: ,Die psychoanalytischen Deutungen werden im Ganzen zur Grimasse eines Lesens der Chifferschrift, wie sie einem glaubenslosen Zeitalter gemäß war. Statt auf die Wirklichkeit transzendenten Seins hin wird auf das seelischunbewußte Geschehen hin gedeutet. Statt Erhellung und Glaube wird scheinerklärung und Aberglaube vollzogen." $\mathrm{Cf}$. auch Bertalanffy $47 \mathrm{ff}$.; besonders Wisse $134 \mathrm{f}$. 
1.2. Großen Einfluß auf das Verständnis des Symbolbegriffs überhaupt und damit auch auf das religiöse Symbol hat Cassirer ausgeübt ${ }^{6}$. Ausgehend von der deutschen idealistischen Philosophie, hat er seine Philosophie der symbolischen Formen entwickelt. Er hat sich hauptsächlich sprachpsychologischer und ethnologischer Materialien bedient. Er hält sich auch konsequent an die zu seiner Zeit herrschende Vorstellung einer Entwicklungsgeschichte. Praktisch wirkt sich das so aus, daß er mit Hilfe umfangreichen Materials zu beweisen sucht, daß auf dem Gebiet der symbolischen Formen eine Entwicklung von einer, ,sinnlich aufgefaßte[n] Wirklichkeit" über eine mythische Weltauffassung zur Religion zu beobachten sei. Die letzte Entwicklungsstufe sei die Religionsphilosophie. Seine Absicht war es, den Dualismus zu überwinden, der zwischen der empirischen und der außerempirischen Welt besteht: ,Immer wieder sind wir im Laufe unserer Untersuchung zu der Ensicht geführt worden, daß der echte und wahrhafte Begriff des 'Symbolischen' sich den herkömmlichen metaphysischen Einteilungen und Dualismen nicht fügt, sondern daß er ihren Rahmen sprengt. Das Symbolische gehört niemals dem 'Diesseits' oder 'Jenseits', dem Gebiet der 'Immanenz' oder 'Transzendenz' an: sondern sein Wert besteht eben darin, daß es diese Gegensätze, die einer metaphysischen Zweiweltentheorie entstammen, überwindet. Es ist nicht das Eine oder das Andere, sondern es stellt das 'Eine im anderen' und das 'Andere im Einen' dar. So konstituiert die Sprache, der Mythos, die Kunst je ein selbständiges und charakteristisches Gefüge, das seinen Wert nicht dadurch erhält, daß in ihm ein äußeres und jenseitiges Dasein irgendwie 'abgespiegelt' erscheint. Ihr Gehalt wird ihnen vielmehr dadurch zuteil, daß sie, je nach einem eigenen ihnen innewohnenden Bildungsgesetz, eine eigentümliche und selbständige, in sich geschlossene Welt des Sinnes aufbauen." 7

Mit seinen Vorstellungen schließt sich Cassirer der ,Erkenntnislehre“ der Neukantianer $a^{8}{ }^{8}$. Der tiefe Eindruck, den die erstgenannte Schule hinterlassen hat, ist bei der sogenannten Eranos-Gruppe (Eliade, Kerény u.a.) zu erkennen, während die letztgenannte viele theologisch orientierte Denker (Bultmann, Wach, Tillich $u$. a.) beeinflußt hat.

${ }^{6}$ Sein dreibändiges Werk, ,,Die Philosophie der symbolischen Formen", erschien bereits 1923-1929. Auf den Einfluß von Cassirers Gedanken auf andere Forscher (Plachte, Urban) hat Looff, 44, hingewiesen, der auch vom Standpunkt der protestantischen Theologie Kritik geäußert hat ( $96 \mathrm{ff}$.).

7 Cassirer 3,404f. Goodman, 228, hat erkannt, daß auch die heutige positivistische Philosophie die symbolischen Ausdrucksformen auf eine frühere Entwicklungsstufe verweist: ,[...] these were ages of myth, when people living in a fearful and uncon- trollable environment could not distinguish magic and science, nor saga and history, nor dream and empirical experience; the poets were the prophets, historians, philosophers, and scientists. In the course of time, poetry was replaced by philosophy and history; and these in turn have given way to special physical sciences and positivist sociology. In our time, literature can be merely decoration or entertainment or exercise in emotional noises."

${ }^{8}$ Cf. Bertalanffy 43. 
2. Bedeutungsvoller und fruchtbarer ist es jedoch, sich mit jenen Standpunkten und Begriffsdefinitionen bekannt zu machen, welche immer noch die heutigen Diskussionen über das Wesen des religiösen Symbols, seine Struktur und seine Funktionen bestimmen. Verständlicherweise herrschen gerade in der Frage der Definition des Symbols größte Unklarheiten und Widersprüche ${ }^{9}$. Es ist nicht meine Aufgabe, diese hier im einzelnen darzustellen. Damit aber die im folgenden vorgetragenen Gedanken besser verständlich sind, müssen hier dennoch ein paar früher geäußerte Definitionen des Symbols erwähnt werden.

2.1. Bis in die letzte Zeit dominieren in Diskussionen über das religiöse Symbol die von Tillich geäußerten Gedanken, mit denen er bereits 1928 hervorgetreten ist ${ }^{10}$. Seine Ansicht ist auch deshalb bedeutsam, weil sie die kirchliche Theologie stark beeinflußt hat. Diese Theorie ist aus einem gewissen Gegensatz zu Cassirers kritischem Idealismus erwachsen. Tillich charakterisiert die Struktur des Symbols durch vier Merkmale: ,,Das erste und grundlegende Merkmal des Symbols ist die Uneigentlichkeit. Sie besagt, daß der innere Akt, der sich auf das Symbol richtet, nicht das Symbol meint, sondern das in ihm Symbolisierte. [...]

Das zweite Merkmal des Symbols ist die Anschaulichkeit. Sie besagt, daß ein wesensmäßig Unanschauliches, Ideelles oder Transzendentes im Symbol zur Anschauung und damit zur Gegenständlichkeit gebracht wird. Die Anschaulichkeit braucht keine sinnliche zu sein. Sie kann ebensogut eine vorgestellte sein. [...]

Das dritte Merkmal des Symbols ist die Selbstmächtigkeit. Sie besagt, daß das Symbol eine ihm selbst innewohnende Macht hat, die es von dem bloßen in sich ohnmächtigen Zeichen unterscheidet. Dieses Merkmal ist maßgebend für die Trennung von Zeichen und Symbol. Das Zeichen ist willkürlich austauschbar. Es hat keine Notwendigkeit, weil es keine innere Macht hat. Das Symbol hat Notwendigkeit. Es kann nicht ausgetauscht werden. Es kann nur verschwinden durch Verlust seiner inneren Mächtigkeit, durch Symbolzerfall. Und es kann nicht erfunden, sondern nur geschaffen werden. [...]

Das vierte Merkmal des Symbols ist die Anerkanntheit. Sie besagt, daß das Symbol sozial eingebettet und getragen ist. Es ist also nicht so, daß eine Sache erst Symbol ist und dann Anerkennung findet, sondern Symbolwerdung und Anerkennung gehören zusammen. Der symbolschaffende Akt ist ein Sozialakt, auch wenn er in einem Einzelnen zuerst durchbricht. Der Einzelne kann sich Zeichen machen für seine privaten Bedürfnisse;

\footnotetext{
${ }^{9}$ Skorupski $117 \mathrm{ff}$; Wisse 3, $21 \mathrm{f}$; Urban $580 \mathrm{ff}$; d dazu Firth $50 \mathrm{ff}$.

${ }^{10}$ Hier und im folgenden habe ich die Ausgabe seiner Gesammelten Werke (von 1964) benutzt, in der in unveränderter Form sein Aufsatz „Das religiöse Symbol“ abgedruckt
}

ist, der zum ersten Male in: ,Blätter für deutsche Philosophie" 1 (1928), $277 \mathrm{ff}$., erschien. Er wurde wiederholt in deutscher und englischer Sprache in verschiedenen Ausgaben veröffentlicht. 
Symbole kann er nicht machen; wird ihm etwas zum Symbol, so immer im Hinblick auf die Gemeinschaft, die sich darin wiedererkennen kann. [...]

Diese allgemeinen Merkmale des Symbols gelten - wie die Einzelbeispiele zeigten - auch für die religiösen Symbole. Die religiösen Symbole sind vor den übrigen dadurch ausgezeichnet, daß sie Veranschaulichung dessen sind, was die Sphäre der Anschauung unbedingt übersteigt, des im religiösen Akt Letztgemeinten, des Unbedingt-Transzendenten. [...]

Die religiösen Symbole sind weder gegenständlich, noch geistig-sinnhaft fundiert, sie sind unfundiert, religiös gesprochen, sie sind Gegenstand des Glaubens. Sie haben kein anderes Recht als das der Vertretung des Unanschaubar-Transzendenten, das ihrer nicht bedarf, um zur Existenz zu kommen. Auf dieser Tatsache beruht die eigentümliche Zweischichtigkeit der religiösen Symbole."11

Kurz gesagt, Tillich behauptet, daß das Symbolbewußtsein nicht im Symbol selbst verwirklicht sei, sondern in dem, was damit symbolisiert werde. Durch das Symbol werde das Unsichtbare sichtbar gemacht. Ferner besitzte das Symbol selbst eine bestimmte Kraft, die aber den symbolischen Zeichen fehle. Wie bereits gesagt, hat Tillichs Symbolbegriff das Symbolverständnis der Theologie stark beeinflußt. Dagegen wurden jedoch Einwendungen erhoben, sowohl von der empirisch ausgerichteten Religionswissenschaft als auch besonders von seiten der orthodoxen Theologie ${ }^{12}$.

2.2. In unserer Darstellung des Symbols wollen wir uns nun auch den Definitionen des Symbols durch Mensching zuwenden. Wie mir scheint, erweitern diese den Begriff Symbol bedeutend und helfen, den von Tillich dogmatisch eingeschränkten Begriff ,,gläubiger Realismus" zu überwinden. Mensching hat sich wie folgt geäußert: ,,Symbol ist alles, was zu einer von sich selbst verschiedenen Wirklichkeit in einem sachlich notwendigen Verhältnis der Repräsentation steht, wobei das Repräsentierte je nach Art des Symbols in eine verschiedene Nähe zum Symbol tritt.“

$\mathrm{Zu}$ dieser Definition hat er folgende Erklärungen geliefert:

,,a) 'Symbol ist alles ...': mit diesem Satze ist bereits a priori gesagt, daß der Symbolcharakter einer Sache nicht auf bestimmte konkrete Dinge beschränkt ist, sondern daß prinzipiell alles zum Symbol werden kann, sofern es den in der weiteren Formel angegebenen Bedingungen genügt. Der Symbolcharakter also liegt nicht in irgendeiner Sache an sich, sondern in gewissen zu untersuchenden Beziehungen dieser Sache zu etwas Anderem. [...]

b) 'was zu einer von sich selbst verschiedenen Wirklichkeit ...': das ist die zum Symbolcharakter einer Seinsform notwendige andere Größe. Diese

11 Tillich 1964, 196ff. Manchmal spricht er von 5 Merkmalen (cf. 1973, 5). Royce, 20, hat bei Tillich 6 solcher Merkmale festgestellt.
12 Dagegen werden Einwände, auch von seiten der Psychologen, erhoben, z. B. Royce $19 \mathrm{ff}$. 
andere Größe nennen wir die vom Symbol gemeinte ,Wirklichkeit". Dabei ist natürlich festzustellen, daß unter ,Wirklichkeit" die verschiedenen Möglichkeiten der Realität und nicht etwa nur die gewöhnlich als Wirklichkeit bezeichnete konkrete Welt sinnlicher Erfahrung zu verstehen ist.

c) 'in einem sachlich notwendigen Verhältnis der Repräsentation steht ...': Die Beziehung zu jener anderen Wirklichkeit ist nach zwei Seiten hin zu charakterisieren. Einerseits muß das Verhältnis sachlich notwendig sein. Auf dieses Moment der Notwendigkeit, und zwar einer aus der Sache heraus und nicht durch Zufall gestifteten Notwendigkeit ist das größte Gewicht zu legen. [...] Und das zweite charakterisierende Moment ist die Bezeichnung des Verhältnisses von Symbol und gemeinter Wirklichkeit als Repräsentation.

d) 'wobei das Repräsentierte je nach Art des Symbols in eine verschiedene Nähe zum Symbol tritt.' Die Repräsentation der gemeinten Wirklichkeit durch das Symbol ist in den verschiedenen Arten möglicher Symbolbegriffe nicht von gleicher Art. Es wird sich zeigen, daß eine wesentliche Differenzierung der Symbolarten gerade darin besteht, daß die gemeinte Wirklichkeit mehr oder weniger nahe an das Symbol heranrückt, ja gelegentlich selbst in das konkrete Symbol eintritt." "13

Menschings Gedanken sind kurz gefaßt folgende: Symbol kann jede Erscheinung sein, die notwendig eine andere von dieser unterschiedliche Erscheinung vertritt. Mit diesen wenigen Beispielen aus der gegenwärtigen Diskussion über das Symbol sind wir bei der Wesensfrage angelangt ${ }^{14}$.

3. Diskussionen darüber, warum der Mensch Symbole benutzt, sind überflüssig, insbesondere deshalb, weil man auf diese Frage nicht genetisch antworten kann. Man kann zwar vermuten, weshalb der Mensch des Neolithikums die elementarsten geometrischen Zeichen - Punkte und Striche - oder auch komplizierte geometrische Muster in die Wände seiner Höhlen eingraviert hat, man kann aber nicht einmal mit gewisser Sicherheit sagen, ob er das aus spielerischer Freude getan hat oder ob damit auch irgendwelche religiöse Erlebnisse und Ideen verbunden waren. Die Genese des Gebrauchs von Symbolen ist nicht aufzuklären ${ }^{15}$. Um sagen zu können, ob diese Zeichen einen Sinn haben, müßte man wissen, zu welchem Zweck

13 Mensching 1930, 1070. Er ist später noch einmal auf den Begriff und die Struktur des Symbols zurückgekommen: ,,Symbol ist alles, was für ein Subjekt zu einer von sich selbst verschiedenen im Symbol gemeinten Sinnwirklichkeit in ein Verhältnis der Repräsentation gesetzt wird“" $(1955,362)$.

14 Zum Vergleich können wir hier auf verschiedene andere Definitionen hinweisen: in der Philosophie auf Seiffert 25 f.; Friedmann 29; in der Soziologie auf Parsons 416; ThassThienemann 17ff; in der Psychologie auf Jaspers 1947, 1032; 1956, 150.
${ }_{15}$ Das wird mehrfach vermerkt. So Langer, Susanne 41: , This basic need, which certainly is obvious only in man, is the need of symbelization. The symbol-making function is one of man's primary activities, like eating, looking, or moving about. It is the fundamental process of his mind, and goes on all the time. Sometimes we are aware of it, sometimes we merely find its results, and realize that certain experiences have passed through our brains and have been digested there." Ähnlich auch Mensching 1955, 362; Giedion 87. 
sie angebracht wurden. Dies wird deutlicher bei der Betrachtung der Funktionen von Sprachzeichen. Es ist wahr, daß z. B. im geschriebenen Wort „Berg" vier verschiedene Zeichen zu sehen sind, in der Aussprache dieses Wortes dagegen hören wir vier Laute verschiedenen Klanges. Weder die geschriebenen Zeichen noch die hörbaren Laute haben alle zusammen oder einzeln irgendeine symbolische Bedeutung. Sie können als Sinnwirklichkeit von Menschen verschiedener ethnischer Gruppen und Kulturen erkannt und gehört werden, ohne daß sich deshalb mit dieser Sinnwirklichkeit die Vorstellung von einem Berg assoziieren würde, d. h. eine andere Sinnwirklichkeit, die diese Zeichen und Laute symbolisieren. Aber nur für die Menschen und die Gemeinschaft, welche einer besonderen Kultureinheit angehören, die die deutsche Sprache versteht, werden diese Zeichen und Laute symbolisch, d.h. sie erhalten einen Sinninhalt ${ }^{16}$. Dieses einfache Beispiel bestätigt das vorhin Gesagte, daß ein Zeichen für sich allein kein Symbol bedeutet, und es zeigt auch, daß die genetische Frage als solche für die Problematik des Gebrauchs von Symbolen nicht wichtig ist. Wichtiger ist es, das Symbol in seiner Struktur und seinen Funktionen zu erkennen.

3.1. Wenn wir uns zunächst der Frage der Symbolstruktur zuwenden, so sollten wir uns daran erinnern, daß die bisherige Forschung besonders den dualistischen - ich möchte lieber sagen: den bipolaren - Charakter des Symbolbegriffs betont hat, wie mir scheint, mit Recht. Das Symbol umfaßt immer sowohl die zu symbolisierende Wirklichkeit als auch die Wirklichkeit des Symbolisierenden. Doch verwirrt sich diese grundlegende, an sich klare Selbstverständlichkeit in dem Moment, wenn man versucht, die Beziehungen dieser beiden Strukturelemente zu klären. Es handelt sich um ein Problem, das viele heftige Diskussionen hervorgerufen hat.

Wiederholt wurde behauptet, daß alles zum Symbol für alles werden könne. Formal ist diese Behauptung richtig, soweit sie rein theoretisch bleibt. Doch zeigt die empirische Erfahrung, daß die Möglichkeiten prak-

${ }^{16}$ Cf. bei Segerstedt das Kapitel ,,Der Laut wird zum Symbol" ( $26 \mathrm{ff}$., besonders 38 und 43). Die Sinngebung des Wortes faßt er in folgender Weise auf: ,Wir fassen das Ergebnis dahin zusammen, daß ein Wort keinen Sinn bekommen kann, insofern es nicht in eine allgemeine Situation eingesetzt wird. Hingegen wird das Wort zum Symbol, weil es auf ein System gesellschaftlicher Normen hinweist, die das Wort teils mit der allgemeinen Situation verbinden, teils aus dieser allgemeinen Situation herausgreifen. Wörter, die als Teile des Ganzen zu Repräsentanten des Ganzen werden, sind Symbole des Ganzen. Wenn wir sagen, die Wörter seien Symbole, so ist es dieses Ganze, dessen Symbole sie sind. Wir sagten also nicht, die Wörter ständen als Symbole des Gedankens an das Objekt und auch nicht als Symbole des Objekts. Wir sagten nicht, ein Mensch sähe zuerst einen Gegenstand, zum Beispiel einen Stuhl, darauf denke er Stuhl und suche nun ein Symbol für seinen Gedanken Stuhl, das er endlich im Worte Stuhl findet. Das Wort ist nach unserer Auffassung Symbol für eine Reihe von Verhalten, die zu einer allgemeinen Situation vereinigt sind. Daß gerade ein bestimmtes Wort verwendet wird, beruht auf einem System gesellschaftlicher Normen, so wie auch unser Verhalten im übrigen auf einem System von Normen beruht" (57). 
tisch sehr eingeschränkt sind ${ }^{17}$. Schon allein die Tatsache, daß erfahrungsgemäß niemals alles zur Symbolisierung von allem verwendet wurde, läßt uns aufmerken. Es ist nur die Frage, was dafür bestimmend ist, daß die Zahl der benutzten Symbole sehr klein ist. Das umfangreiche religionsgeschichtliche Material zeigt unmißverständlich, daß die Auswahl von Symbolen festgelegt und historisch gebunden ist. Darüber braucht man sich nicht zu streiten. Es ist nur die Frage, wodurch diese Auswahl bestimmt wird. Die Antworten können sehr verschieden sein. Die einen behaupten, daß die freie Wahl des Subjekts und dessen Ziel, das mit der Symbolisierung erreicht werden soll, bestimmend seien. Die anderen behaupten, die soziale Struktur, der das Individuum angehört, sei maßgebend für die Auswahl. Die Funktionalisten meinen, daß alle psychischen Aktivitäten von dem Wunsch nach Sicherung der Stabilität der sozialen Struktur motiviert $\operatorname{seien}^{18}$. Ohne sich in diese breite Diskussion einzulassen, kann man sagen, daß man es im einen wie im anderen Fall mit einer Auswahl zu tun hat. Zum Verständnis des Wesens des Symbols trägt die Behauptung wenig bei, daß alles zum Symbol für alles werden könne. Das Symbol ist das Ergebnis einer Auswahl. Daher ist zum richtigen Verständnis seiner Struktur die Frage der Auswahl wichtig.

Auch Mensching hat die Wichtigkeit dieser Frage erkannt. Er hat versucht, darauf mit dem Hinweis zu antworten, wie wir bereits gelesen haben, daß zwischen dem zu Symbolisierenden und dem Symbolisierenden ,ein sachlich notwendiges Verhältnis" bestehe. Diese Antwort ist formal richtig, denn es ist nicht anzunehmen, daß die Auswahl des Symbols nur auf Zufall oder rationaler Willkür beruht, ohne die Fähigkeit, Wesentliches auszusagen, d.h. die zu symbolisierende Wirklichkeit zu repräsentieren. Aber das genügt nicht, denn die folgende Frage lautet: Wer bestimmt, was ,,sachlich notwendig" ist? Mit anderen Worten: von wem hängt es ab, daß ein besonderes Symbol mit etwas Bestimmtem, was symbolisiert werden soll, verbunden wird? Daß diese Frage angebracht ist, ist aus den Fällen zu erkennen, in denen ein und dieselbe zu symbolisierende Erscheinung in verschiedenen Religionen unterschiedliche Symbole erhält. Im germanischen Fruchtbarkeitskult symbolisieren der Eber bzw. die Sau die Fruchtbarkeit, in verschiedenen Gegenden Kleinasiens ist es die Frau mit vielen Brüsten (Artemis von Ephesus), im Hinduismus Schiwa mit vielen Armen. Warum so viele verschiedene Symbole zur Darstellung ein und desselben religiösen Phänomens - der Fruchtbarkeit - geschaffen wurden, ist schwer mit ,,sachlicher Notwendigkeit“ zu erklären, wenn man nicht weitere Gründe aufzeigt, die dafür maßgebend sind. Das erwähnte Beispiel läßt vermuten,

17 Dazu hat sich bereits Saussure, 103, bekannt: ,Le symbole a pour caractère de n'être jamais tout à fait arbitraire; il n'est pas vide, il y a un rudiment de lieu naturel entre le signifiant et le signifié. Le symbole de la justice, la balance, ne pourait pas être remplacé par n'importe quoi, un char, par exemple."

18 Ausführlicher habe ich mich dazu, 1976, $19 \mathrm{ff}$, geäußert. 
daß die Auswahl des Symbols von dem zu symbolisierenden Gegenstand unabhängig ist. Hier mag noch an einige symbolische Darstellungsweisen der hl. Maria erinnert werden: den Stern, die Rose, die Hirtin u. v. a. Es gibt nur ein Wesen Maria, aber es wird verschieden symbolisiert. Vielleicht möchte man hier ergänzen, es sei damit zu erklären, daß Maria in verschiedenen Funktionen auftrete, die später die Auswahl der Symbole bestimmt hätten. Dann könne man sagen, daß abhängig davon, in welchen Funktionen sie erscheint, die entsprechenden Symbole in ,,sachlicher Notwendigkeit" ausgewählt worden seien. Doch diese Ergänzung bestätigt gerade das, was ich sagen wollte: Nicht das zu symbolisierende Objekt die hl. Maria - ist diejenige, die sich in verschiedenen funktionellen Situationen befindet, sondern das Subjekt. In Übereinstimmung damit wählt es das entsprechende Symbol, das ihm in dieser Situation ,,sachlich notwendig“ erscheint. Man könnte noch weitergehen und die funktionelle Situation analysieren und damit deren Struktur bestimmen. Dadurch könnten die Motive des Subjekts für die Auswahl von Symbolen noch klarer werden. Hier wollen wir uns auf die Feststellung beschränken, daß die Auswahl des Symbols von der funktionellen Situation des Individuums abhängig ist. Mit anderen Worten, die Auswahl des Symbols ist stets durch seine Subjektbezogenheit bestimmt.

Aus dieser Erkenntnis ergibt sich, daß die These, alles könne für alles zum Symbol werden, auf die konkret gegebene Subjektbezogenheit einzuschränken ist. Wenn man die Möglichkeiten zur Auswahl von Symbolen in der Weise erweitern würde, daß diese Auswahl durch das zu symbolisierende Objekt bestimmt oder zumindest mitbestimmt wird, dann wäre die These von der prinzipiellen Möglichkeit, alles könne durch alles symbolisiert werden, nicht aufrecht zu erhalten. Der Vorgang der Symbolisierung ist einseitig. In verschiedenen Religionen kennt man eine Sonnensymbolik - vom einfachen geometrischen Kreis bis zu so ausdrucksvollen Gestalten wie einer schönen Frau und einem bärtigen König. Dadurch wird ein übriges Mal bestätigt, daß es nicht das Objekt des religiösen Erlebnisses ist ${ }^{19}$, sondern dessen Träger, das Subjekt, das die Auswahl des Symbols trifft.

3.2. Aus dem Gesagten wird deutlich, daß damit die anfangs angesprochene Frage des bipolaren Charakters des Symbols aktualisiert wird. Es handelt sich um die Frage nach der empirischen Ausdrucksform des Symbols und dessen transzendentem Inhalt. Die Behauptung, daß das empirisch gegebene religiöse Symbol immer eine nichtempirische Wirklichkeit darstelle, ist schon fast zum Axiom geworden. Erinnern wir uns hier nur an die im vorangegangenen Zitat wiedergegebenen Gedanken von Tillich: ,,Die religiösen Symbole sind vor den übrigen dadurch ausgezeichnet, daß sie Veranschaulichung dessen sind, was die Sphäre der Anschauung unbedingt übersteigt, des im religiösen Akt Letztgemeinten, des Unbedingt-Trans-

${ }^{19}$ Cf. Fawcett 33. 
zendenten."20 Das Unbedingt-Transzendente ist verstanden als Gott. Mit besonderem Pathos hat Leese das betont: ,Religiöse Symbole sind nicht willkürliche Zeichen, die man nach Belieben erfinden und deren Bedeutung man auf dem Wege der Verabredung festsetzen könnte (Allegorie), religiöse Symbole sind nicht abstrakte Schemata, die in keinem inneren Verhältnis zu dem stehen, worauf sie hinweisen, religiöse Symbole sind dem menschlichen Zugriff weitgehend entzogene Wesenheiten (essentiae), die mit einer das Gefühl tief erregenden Seinsmächtigkeit geladen sind, die dem Ungeheuren rufen, weil dieses sich in ihnen offenbart. [. . .] Für ein religiöses Symbol sind zwei Voraussetzungen konstitutiv: eine erlebende Seele und ein zu erlebendes Göttliches. Das religiöse Symbol selbst stellt mit seiner Dialektik von Eigentlichkeit und Uneigentlichkeit, von Anschaulichkeit und Unanschaulichkeit, von Sinnlichkeit und Unsinnlichkeit, von Immanenz und Transzendenz die Mittlerschaft, den Kontakt zwischen beiden her." 21

Leeses Worte enthüllen, daß er von einer sachlichen Darstellung des Wesens des Symbols zu einem Bekenntnis seiner religiösen Überzeugung übergewechselt ist. Das Symbol ist für ihn zu einer Aussage über die göttliche Offenbarung geworden. Wenn wir diesen Teil seiner Äußerungen beiseite lassen, wird jedoch deutlich, daß er das Problem der Bipolarität ganz richtig erkannt hat. Diese ist das Grundmerkmal der Struktur des religiösen Symbols. Es will immer mit ,,irgend etwas" ,,irgend etwas" anderes aussagen. Damit ist das Problem jedoch nicht erschöpft.

Ist diese Bipolarität ontologisch oder psychologisch bestimmt? Daß das „Unbedingt-Transzendente“ (Tillich) und,,entzogene Wesenheiten (essentiae)" (Leese) ontologisch zu verstehen sind, ist außer Zweifel. Es ist die transzendente Wirklichkeit, die durch den Akt der religiösen Symbolisierung in die Sinnwirklichkeit übertragen wird. Diese ontologische Bipolarität bestimmt das Verständnis des theologischen Symbols. Es ist nur die Frage, ob sie aufrecht erhalten werden kann. Daß es mit dem ontologischen Verständnis seine Schwierigkeiten hat, wurde, wie es scheint, bereits von Mensching zugegeben, wie seine Überlegungen über die Beziehungen der symbolisierenden Wirklichkeit zum Symbol zeigten. In diesem Zusammenhang spricht er von ,,verschiedener Nähe zum Symbol“* und ,,daß die gemeinte Wirklichkeit mehr oder weniger nahe an das Symbol herantritt" "Schon dieser Gedanke der verschiedenen Entfernung beider Sphären ist bezeichnend. Sein Gedankengang mündet konsequenterweise in der Behauptung, daß die zu symbolisierende Wirklichkeit ,,gelegentlich selbst in das konkrete Symbol eintritt". Diese Aussage zeigt, daß die Bipolarität zwischen dem zu Symbolisierenden und dem Symbolisierenden in

20 Tillich 1964, 197. Ebenso erwähnt auch 21 Leese 1934, 10. 
einer gewissen Situation verschwunden ist. An ihre Stelle ist zweifellos die Identität getreten. In diesem Fall handelt es sich um ein Erlebnis von anderer Qualität, nämlich um ein direktes, undifferenziertes religiöses Erlebnis. Das ist dann kein Symbolisierungsakt mehr ${ }^{22}$. Das hat auch Kahler erkannt: , Religious and artistic imagery arose in common. Earliest images, prehistoric cave paintings, are not symbols as yet, they are virtual acts of seizure; they do not signify or represent, they actually are the creatures represented. They do not point to prototypes, they are pointed at with the points of arrows. Similarly, the original totemistic idol, as long as the deity is believed to be actually present in the image, is not a likeness of the worshipped being, it is the being itself. Only when a difference is felt between the visually present idol and a remote, or temporarily absent, deity, when the image turns into a mere residue or residence of the deity, only then does the image become a symbol." "23

Dieses Hineingleiten in die Identität, wobei der Symbolisierende völlig mit dem zu Symbolisierenden übereinstimmt, zeigt, daß diese Möglichkeit der Struktur des Symbols widerspricht. Die Bipolarität ist daher als wesentliches Kennzeichen der Struktur des Symbols anzusehen ${ }^{24}$.

Einerseits haben wir bereits vorhin auf die Schwierigkeiten bezüglich der ontologischen Bipolarität hingewiesen, da sie im faktischen Symbolisierungsakt empirisch nicht zu begründen ist. Andererseits aber sagten wir soeben, daß die Bipolarität wesensmäßig zur Symbolstruktur gehöre. Dieser scheinbare Widerspruch läßt die Bipolarität des Symbols unter einem anderen Aspekt betrachten. Der wahre Grund, weshalb die These von der ontologischen Bipolarität nicht aufrecht erhalten werden kann, liegt im Ge-

${ }^{22}$ Hier kann man auf eine Diskussion hinweisen, die manchmal recht scharfe Formen angenommen hat, nämlich darüber, ob der Himmel mit Gott identisch ist oder ob der Himmel nur eine Ausdrucksform für Gott (ein Symbol) ist. Darüber habe ich mich, 1961, $20 \mathrm{ff}$., ausführlicher geäußert. Mir scheint, daß Giedion, 87, den Zusammenhang zwischen Symbol und Realität beim prähistorischen Menschen und die spätere Entwicklung richtig erkannt hat: , The essential nature of the symbol has always consisted in this urge to express the inherently inexpressible, but in primitive times the crystallization of a concept in the form of a symbol portrayed reality before that reality came to pass. The symbol itself was reality [kursiv von $\mathrm{H}$. B.], for it was believed to possess the power of working magic, and thus of directly affecting the course of events: the wish, the prayer, or the spell to be fulfilled. Herein lies the contrast between the function of the symbol in prehistory and in later periods. In
Greece the symbol was not only a means of recognition. It also developed a spiritual content and became an abstract concept. It was not an independent agent." Es dürfte darüber kein Zweifel bestehen, daß mit Dyaus, Jupiter u. a. anfangs der Himmelsgott selbst bezeichnet wurde, aber ebenso zweifellos ist der Himmel erst auf einer späteren Stufe der Abstraktion, und nicht nur in der christlichen Theologie, sondern auch in anderen religiösen Systemen (Brahmaismus, Buddhismus), zum Aufenthalt Gottes oder zu dessen Erscheinungsform geworden, also zu einem Symbol.

${ }^{23}$ Kahler 61.

${ }^{24}$ Sehr bestimmt wird dieser Gedanke auch von Seiffert, 27, gestützt: ,,Die Dualität, die bezeichnend ist für das Sinnbild, kann auf keine Weise eliminiert werden." Ein andermal spricht er von ,ZWwiegesichtigkeit" (9). Ungeachtet seines Bestrebens, die Bipolarität $\mathrm{zu}$ überwinden, ist Jaspers gezwungen, von ,Zweideutigkeit“" zu sprechen $(1946,277)$. 
brauch des Begriffs ,,Wirklichkeit". Im einen Fall ist die Rede von der Sinnwirklichkeit (vom Symbolisierenden bzw. vom Symbol), im anderen Fall handelt es sich um das ,,Letzt-Wirkliche“" (das Religiöse, Gott). Solch eine attributive Bestimmung des Wirklichkeitsbegriffs kann jedoch keine größere Klarheit bringen, da es bei der Frage hier nicht um die Wirklichkeit als solche geht. In beiden Fällen ist die Rede von einer subjektbezogenen Wirklichkeit. Es ist das Subjekt, das in seiner psychischen Aktivität im Falle der religiösen Symbolisierung in seinem religiösen Leben den Inhalt seines Erlebnisses durch etwas Anderes indirekt ausdrückt. Wenn man unbedingt den Begriff der Bipolarität gebrauchen möchte, dann muß man ihm in diesem Fall im Gegensatz zur ontologischen Bipolarität einen anderen Inhalt geben. Dann müßte man von einer psychologischen Bipolarität sprechen. Hier ist zu bemerken, daß die Übertragung des Symbolinhalts von der ontologischen auf die psychologische Ebene nichts an der formalen Struktur des Symbolbegriffs ändert, d.h. deren Bipolarität weder einschränkt noch aufhebt.

3.3. Obgleich ich mich zum bipolaren Charakter der Symbolstruktur bekannt habe, meine ich doch, daß man die Ansichten nicht unbeachtet lassen kann, die seinerzeit versucht haben, diesen bipolaren Charakter zu überwinden, wenn auch mit anderer Begründung. So spricht bereits Cassirer vom ,,Begriff des mit sich selbst identischen Symbolischen“. In letzter Konsequenz möchte er, wie er sagt, den Dualismus überwinden. ,Wir können 'Bedeutung' nicht anders als durch Rückbeziehung auf die 'Anschauung" erfassen - wie uns Anschauliches nie anders als im 'Hinblick' auf Bedeutung 'gegeben' sein kann. Halten wir hieran fest, so entgehen wir damit der Gefahr, daß das Symbolische unserer Erkenntnis sich in sich selber spaltet, daß es gewissermaßen in einen 'immanenten' und einen 'transzendenten' Bestandteil auseinanderbricht. Das Symbolische ist vielmehr Immanenz und Transzendenz in Einem: Sofern in ihm ein prinzipiell überanschaulicher Gehalt in anschaulicher Form sich äußert." 25

Cassirer begründet seine Ansicht mit der Entwicklung der Menschheit. Diese komme von mythischen Vorstellungen, die mit Hilfe von Symbolen ausgedrückt werden, zu immer höherer Entwicklung. Dabei wähle sie, um ihren Vorstellungen Ausdruck zu verleihen, begriffliche Formulierungen. Mit anderen Worten: die Entwicklung gehe vom Konkreten zum Abstrakten. Nur die sinnliche Auffassung der Welt verwende die mythischen und religiösen Symbole. Auf einer höheren Entwicklungsstufe verlören diese religiösen Symbole ihren Ursinn, ihren Zusammenhang mit dem Mythischen und Religiösen. An ihre Stelle würden Begriffsbildungen treten $^{26}$. Diese These, die offensichtlich unter dem seinerzeitigen Einfluß

25 Cassirer 3, 447.

${ }^{26}$ Das hat Looff, 41, richtig erkannt: ,,Mit dieser Symboldeutung wäre das religiöse
Symbol, das seiner Eigenart gemäß doch nie auf einen Hinweis auf etwas außer ihm bzw. über ihm verzichten kann, weil es von der 
der evolutionistischen Ideologie entstanden ist, ist jedoch nicht zu halten. Auch ist es nicht nachprüfbar, daß die mythische Weltanschauung durch ein begriffsmäßiges Denken überwunden wird.

3.4. Der zweite Versuch, die ontologische Bipolarität zu überwinden, ist mit dem Namen Jaspers verbunden. Er ist nach einer anderen philosophischen Konzeption unter starkem Einfluß des Existentialismus, insbesondere nach dem Verständnis von Heidegger, angelegt. Jaspers bekennt sich im Prinzip zur Transzendenz: ,, Sein ist für uns, sofern es im Dasein zur Sprache wird. Ein bloßes Jenseits ist leer und so gut, als ob es nicht wäre. Daher fordert die Möglichkeit der Erfahrung eigentlichen Seins immanente Transzendenz.

Diese Immanenz aber hat einen offenbar paradoxen Charakter. Immanent ist grade in Unterscheidung vom Transzendenten im Bewußtsein überhaupt das für jedermann übereinstimmend Erfahrbare, die Welt. Immanent ist dann die existentielle Gewißheit des Selbstseins, welches zwar keinem Bewußtsein überhaupt mehr zugänglich, aber sich selbst gegenwärtig ist im Unterschied vom Sein der Transzendenz, das für Existenz als das ist, worauf als eigentliches Sein sie sich bezieht. Wird aber das Sein der Transzendenz der Existenz gegenwärtig, so nicht als es selbst - denn es besteht keine Identität von Existenz und Transzendenz - , sondern als Chiffre und auch so nicht als Gegenstand, der dieser Gegenstand ist, sondern gleichsam quer zu aller Gegenständlichkeit. Die immanente Transzendenz ist Immanenz, die sogleich wieder verschwand; sie ist Transzendenz, die im Dasein Sprache als Chiffre wurde. Wie im Bewußtsein überhaupt das Experiment der Mittler zwischen Subjekt und Objekt ist, so die Chiffre zwischen Existenz und Transzendenz." 27

In diesen Sätzen treffen wir auf zwei Begriffe, die eine Erklärung verlangen: „Chiffre" und ,immanente Transzendenz". Die Chiffre ist eine besondere Erscheinungsform der Transzendenz. Das ist als Paradoxon zu verstehen, denn in der Chiffre wird die Transzendenz weder zum Objekt, noch wird das objektiv Seiende zum Subjekt ${ }^{28}$. Solch eine Chiffre ist demnach ein Symbol. Was will Jaspers mit dem ebenso paradoxen Begriff

dialektischen Entgegensetzung von Immanenz und Transzendenz lebt, als einer durch den fortschreitenden Erkenntnisprozeß überwundenen Begriffsform zugehörig erkannt. In der Tat sicht Cassirer die religiöse Entwicklung in der prophetisch-monotheistischen Religion im Sinne einer wachsenden Ablösung von allem Dinglichen." Die gleichen Gedanken werden auch bei Bevan, $295 \mathrm{ff}$., wiedergegeben: ,,So far as the intellectual concept stands for a Reality which differs from it, it is a symbol only. So far as it corresponds with the Reality, it is not a symbol, but the actual truth. All our effort to think true thoughts about God is an effort to get rid of the symbolical character of our conceptions, to change them from symbols into precise apprehensions. And if there has been any progress in thought about God between the primitive level and that of a twentiethcentury philosopher, progress has consisted in freeing conceptions from symbolical imagery." Cf. auch Kahler 55, $64 \mathrm{f}$.

27 Jaspers 1956, $136 \mathrm{f}$.

${ }^{28}$ Der Begriff Chiffre steht in Jaspers" Erkenntnistheorie im Mittelpunkt, und er kommt wiederholt darauf zurück. Cf. auch $1947,33 \mathrm{ff}$. 
,,immanente Transzendenz" ausdrücken? In Wirklichkeit will er damit zwei Dinge ablehnen. Erstens, daß man mit Hilfe des Symbols das Transzendente als seiendes Objekt anerkennen könne. Zweitens, daß das Subjekt die Fähigkeit ,der Wahrnehmung und des Hervorbringens metaphysischer Erfahrung" habe. ,In beiden Fällen würde die unergründliche Dialektik des Chiffreseins aufgehoben. Es bliebe ein Jenseits als Transzendenz und ein Diesseits als empirisches Erleben. [...] Nachdem Transzendenz und Immanenz als das einander schlechthin Andere gedacht sind, müssen sie vielmehr in der Chiffre als immanente Transzendenz ihre gegenwärtige Dialektik für uns werden, wenn nicht Transzendenz versinken soll." ${ }^{29}$ Wie diese Worte deutlich machen, will Jaspers eine Möglichkeit finden, um in seinem System der existentiellen Philosophie auch der Transzendenz einen Platz einzuräumen. Und hier wird nicht nur klar, daß er mit dem Begriff der ,,immanenten Transzendenz" den bipolaren Charakter des Symbols zu überwinden trachtet, sondern auch der Widerspruch dieser Konstruktion zur Grundkonzeption deutlich, dem Subjekt als letztem Deuter des Symbolinhalts, denn diesem ist die Transzendenz in seiner existentiellen Situation nicht zugänglich. ,,Das Sein der Transzendenz an sich ist unabhängig von mir, als solches aber nicht zugänglich. Diese Weise der Zugänglichkeit eignet nur Dingen in der Welt. Von der Transzendenz aber vernehme ich nur soviel, als ich selbst werde; erlahme ich, so trübt sie sich in ihrer an sich steten Gegenwärtigkeit; erlösche ich bis zum Dasein eines bloßen Bewußtseins überhaupt, ist sie verschwunden; erfasse ich sie, so ist sie für mich das Sein, das allein ist und ohne mich bleibt, was es ist." 30

Indem Jaspers seine Stellungnahme in dieser Weise präzisiert, ist er gezwungen, seinen erkenntnistheoretischen Subjektivismus zuzugeben. Davon, ob eine Behauptung der Wahrheit entspreche, überzeuge er sich als Subjekt selbst: ,Ich überzeuge mich durch das, was ich selbst bin und will, nicht durch Verstand und empirische Beobachtung. Der Maßstab ist nicht mehr der einer wissenschaftlichen methodischen Untersuchung mit einem Endergebnis, sondern die Frage ist die nach existentiell wahrer und existentiell ruinöser Chiffresprache. ${ }^{\text {" } 31}$

Sein Versuch, die ontologische Bipolarität mit Hilfe des Begriffs der immanenten Transzendenz zu überwinden, ist lediglich von tendenzieller Bedeutung. Auf diese Weise kann man sie aus erkenntnistheoretischen Gründen nicht überwinden.

Wenn man an der vorhin dargelegten Struktur des Symbols festhält, ist es möglich, den Gedanken der Identität noch weiter zu vertiefen. Die Behauptung von zwei völlig getrennten Elementen - dem Symbolisierenden und dem zu Symbolisierenden - in der Struktur des Symbols zeigt einen nur scheinbaren Widerspruch. Das wird deutlich, wenn man nach dem

29 Jaspers 1956, 137.

31 Ib. 148 .

${ }^{30}$ Ib. 
Wesen der zu symbolisierenden Wirklichkeit fragt. Die Theoretiker der religiösen Symbolik, mit Tillich an der Spitze, betonen besonders die transzendente Wirklichkeit, die mit Hilfe eines Symbols ausgedrückt werde. Das gleiche mit anderen Worten: Es ist die besondere Funktion des religiösen Symbols, dem Menschlichen das Göttliche verständlich zu machen, den Kontakt zwischen Gott und dem Menschen zu festigen ${ }^{32}$.

$\mathrm{Zu}$ diesen Ansichten ist zu bemerken, daß die transzendente Wirklichkeit in religiösem Zusammenhang immer erlebte Wirklichkeit ist. Die Welt des Heiligen hat sich stets im Bewußtsein des Individuums gebildet. Sowohl die zu symbolisierende als die symbolisierende Wirklichkeit sind Strukturelemente ein und desselben religiösen Erlebens. Mit anderen Worten, der Akt der Symbolisierung ist ein synthetischer Vorgang, in dem die ontologische Bipolarität in Form des Erlebnisses überwunden ist ${ }^{33}$. Es genügt, hier darauf hinzuweisen, daß man bei ähnlichen Erlebnissen auf dem Gebiet der Musik, der bildenden Kunst und der Literatur auch nicht von ontologisch bipolaren oder dualistischen Erscheinungen zu sprechen pflegt. Das bedeutet jedoch nicht, wie schon vorhin bemerkt, daß man im Symbolerlebnis nicht einige Strukturelemente aufzeigen könnte, die eine Bipolarität in psychologischer Hinsicht nicht ausschließen.

4. Von den Problemen der religiösen Symbolik steht neben dem Wirklichkeitsproblem auch das Wahrheitsproblem im Mittelpunkt. Das religiöse Symbol hat wie alle Symbole kommunikative Bedeutung. Mit seiner Hilfe wird eine Wahrheit bekanntgemacht. Um seine Funktion zu erfüllen, muß es in Zusammenhang mit der Kultur stehen, in deren Rahmen die Kom-

\footnotetext{
${ }^{32}$ Ich meine, daß das Fußen auf der ontologischen Wirklichkeit in seiner Konsequenz den eigentlichen Symbolisierungsakt unmöglich macht. Das spiegelt sich auch in den bezeichnenden Worten von Tillich, 1964, 212, wider: ,Aber gegen diesen Gedanken, der namentlich in der Gegenwart eine ungeheure Entlastung des religiösen Bewußtseins bedeuten würde, eine Befreiung von der Last fragwürdiger Symbolik, erhebt sich ein schwerwiegender Einwand: Voraussetzung eines unmythischen Redens vom UnbedingtTranszendenten ist die religiöse Möglichkeit, gleichsam durch die Wirklichkeit hindurchzureden. Diese Möglichkeit aber setzt eine in Gott stehende Wirklichkeit voraus, d.h. diese Wirklichkeit ist eschatologisch und nicht gegenwärtig. Für die Gegenwart gilt, daß bestimmte Wirklichkeiten mit Symbolkraft über die anderen gestellt werden müssen und in diesem Darüberstehen Ausdruck dafür sind, daß die Wirklichkeit an sich nicht im Unbedingten steht. Nur sofern das eschaton im Gegenwärtigen als lebendige
}

Macht wirkt, könnte diese Macht zu Worten führen, in denen die Wirklichkeit nicht überschritten ist, sondern in der Wirklichkeit durch die Wirklichkeit hindurchgesprochen wird." Aus diesen unklaren, in paradoxer form ausgedrückten Sätzen wird dennoch eines klar - Tillichs Flucht in die Eschatologie. Damit hat er die Grenzen der kritischen Forschung überschritten und ist in unkontrollierbare Spekulationen hineingeraten. Doch war er dazu gezwungen, um an dem unmöglichen Dualismus von empirischem Symbol und transzendenter Wirklichkeit festzuhalten.

${ }^{33}$ Meinen eigenen Gedanken sehr nahe kommt in diesem Fall Leese 1948, 439: ,Insofern also trägt die Gottesanschauung der Religion des protestantischen Menschen synthetischen Charakter. In ihr wirkt sich letztlich die Dialektik von 'Grund' und 'Abgrund', der 'Analogia Entis' und des 'Ganz Anderen' aus, aber diese Dialektik nicht statisch, sondern dynamisch verstanden." 
munikation stattfindet. Einfacher ausgedrückt, darin besteht der soziale Charakter des Symbols. Wir können hier nicht weiter darauf eingehen, obwohl auch diese Frage von zentraler Bedeutung ist, denn von ihr hängt die Kraft des Symbols $a^{34}$. In den Diskussionen über den Wahrheitsgehalt des Symbols herrscht verhältnismäßig große Einmütigkeit, nämlich insofern, daß die mit Hilfe eines Symbols ausgedrückte Wahrheit sich nicht in rationale Kategorien einreihen läßt. Damit erhebt sich die Frage nach den verschiedenen Begriffen der Wahrheit. Meiner Meinung nach ist dieser Widerspruch wegen seiner prinzipiellen Natur nicht aufzulösen ${ }^{35}$. Man kann nur den verschiedenen Inhalt dieses Begriffs präzisieren. Das hilft, müßige Diskussionen und Gegensätzlichkeiten bezüglich des rational bestimmten und des praktisch verwendeten symbolischen Wahrheitsbegriffs in der Religion zu vermeiden. Ferner hilft es auch, Einblick in die Funktionen des religiösen Symbols zu gewinnen.

Wenn man über die Kraft der Wahrheitsaussage von Symbolen spricht, wird unter anderem behauptet, daß man diese in zwei Typen einteilen könne: in diskursive und nicht-diskursive Symbole. Zu letzteren gehörten die religiösen Symbole ${ }^{36}$. Zur ersten Gruppe würden demnach Symbole gehören, die dazu dienen, die rationale Wahrheit auszudrücken. Diese Einteilung berührt verständlicherweise sehr stark die Religionswissenschaft. Wenn diese darauf Anspruch erheben will, eine Wissenschaft zu sein, kann sie auf den Gebrauch solcher Symbole, wie sie dem diskursiven Denken

${ }^{4}$ Tillich spricht in diesem Zusammenhang von ,,Selbstmächtigkeit“ des Symbols (1964, 196), Giedion von ,,potent symbol" (37); von ,Macht des Wortes" im weiteren Sinne spricht Segerstedt (57).

35 Dieser unausgeglichene Gegensatz der Ansichten wird eindrucksvoll durch folgende einander widersprechende Behauptungen charakterisiert: ,,Das Kriterium der Wahrheit eines Symbols kann natürlich nicht der Vergleich mit der Wirklichkeit sein, auf die es hinweist, wenn diese Wirklichkeit gerade das schlechthin Unfaßbare ist. Die Wahrheit eines Symbols ruht in seiner inneren Notwendigkeit für das symbolschaffende $\mathrm{Be}$ wußtsein. Zweifel an seiner Wahrheit zeigen eine Änderung des Bewußtseins, eine neue Stellung zum Unbedingt-Transzendenten an. Das einzige Kriterium, das überhaupt in Frage kommt, ist dieses, daß das Unbedingte in seiner Unbedingtheit rein erfaßt wird. Ein Symbol, das dieser Anforderung nicht genügt, das ein Bedingtes zur Würde des Unbedingten erhebt, ist zwar nicht unrichtig, aber dämonisch." (Tillich 1964, 208.)

Den entgegengesetzten Standpunkt vertritt Leese 1948, 404: ,,Allein, der Grad der Aus- druckskraft für das Unbedingt-Transzendente, so wichtig er ist, kann unmöglich das alleinige Kriterium für die Wahrheit eines religiösen Symbols sein. Es läge in ihm noch nicht die mindeste Begründung dafür, etwa die christliche Symbolwelt der islamischen oder buddhistischen, der spätjüdischen oder neuplatonistischen mit ihrer starken $\mathrm{Be}$ tonung der göttlichen Transzendenz vorzuziehen."

Indem er sich zum ontologischen Charakter des Symbols bekennt, erklärt Wisse seine ausgesprochen theologische Position und begründet sie in der Offenbarung (180f.). Das hat den Wert einer objektiven Erkenntnis (229ff.). Natürlich bringt diese Einstellung nichts Wesentliches in die Diskussion über das Symbol, sondern stellt lediglich Wisses gläubiges Bekenntnis dar.

${ }^{36}$ Cf. Bertalanffy $361 \mathrm{ff}$. Auch Langer, Susanne 97. Sie benutzt anstelle des Terminus ,,nicht-diskursiv" den Ausdruck ,,presentational symbolism", doch mit der gleichen $\mathrm{Ab}$ sicht, die Unterscheidung verschiedener Kategorien von Symbolen zu ermöglichen. Cf. auch Fingesten 150. 
bekannt sind, nicht verzichten. Das wird auch von der Theologie bestätigt, die, wenn sie auch von gewissen Annahmen ausgeht, dennoch das System ihrer Erkenntnisse im Einklang mit den Prinzipien des diskursiven Denkens bildet. In dieser Hinsicht unterscheidet sie sich nicht von der Mathematik. $\mathrm{Daß}$ es sich so verhält, zeigen sehr anschaulich die Versuche der Religionsphilosophie, unter dem Einfluß der analytischen. Philosophie die Funktionen Gottes mit Hilfe mathematischer Formeln auszudrücken. Gleichzeitig aber zeigen diese Versuche die tiefe Kluft zwischen den durch mathematische Formeln ausgedrückten Funktionen Gottes und dem im praktischen religiösen Leben empfundenen Gott. Das weist ein weiteres Mal auf die Notwendigkeit hin, von verschiedenen Symboltypen zu sprechen, wenn auch nur von den vorhin erwähnten diskursiven und nicht-diskursiven Symbolen. Das bedeutet, daß die im diskursiven Denken und damit auch von der Theologie benutzten Symbole sich ihrem Wesen nach von den Symbolen unterscheiden, wie sie in der Religion, der Kunst, der Literatur usw. anzutreffen sind. Um diesen Unterschied zu betonen, pflegt man in letzter Zeit die im diskursiven Denken verwendeten Symbole Zeichen zu nennen ${ }^{37}$.

4.1. Die Ausdrücke ,Zeichen" und ,Symbol" verhelfen zu größerer Klarheit im Gebrauch des Symbolbegriffs; aus diesem Grunde betrachten wir sie hier näher. Vielfach wurde bereits darauf hingewiesen, daß auch in der Forschung nicht selten Ausdrücke der Umgangssprache benutzt werden, die vieldeutig sind. So ist es besonders in der Praxis mit diesen beiden Begriffen geschehen. Das hat auch die sachliche Darstellung des religiösen Symbolbegriffs sehr erschwert. ,Das Wort Symbol hat im Sprachgebrauch einen vielfachen Sinn. Es wird im weitesten Sinn gebraucht für bloße Zeichen, für Gleichnisse und Vergleiche in der Welt, für Schemata und Abkürzungen der Anschauung, für alles Bedeutungshafte. Es ist jeweils zu fragen: Symbol wofür? Ist eine Antwort durch einen Gegenstand in der Welt möglich, so handelt es sich um kein eigentliches Symbol. Das 'wofür' ist hier nur im Symbol selber da und außerdem kein Gegenstand, es sei denn in transzendierenden philosophischen Begriffen." ${ }^{38}$

Hier wird auf die bedeutungsvollste Eigenschaft des Ausdrucks ,Zeichen" eingegangen, die auch beim Gebrauch dieses Begriffs zu beachten ist. Von ,Zeichen" kann man in den Fällen sprechen, wenn damit ein Gegenstand (empirisch oder ideell gegeben) bezeichnet werden soll ${ }^{39}$. In Wirklich-

${ }^{37}$ Die im diskursiven Denken verwendeten Symbole sind in Wirklichkeit frei gewählte Zeichen, die nach Belieben austauschbar sind. Ähnlich ist es in der Theologie, deren Symbole Zeichen bzw. Hilfsmittel sind, um den Inhalt der Religion in Formen des diskursiven Denkens auszudrücken. Die Probleme, die mit dem Gebrauch von ,Zeichen" und ,Symbolen" verbunden sind, haben seit Saussure, 103, die Gedanken der Wissen- schaftler, insbesondere auf dem Gebiet der strukturellen Linguistik und der analytischen Philosophie, stark beschäftigt. Unter deren Einfluß, insbesondere dank Levy-Strauss, ist daher auch ein Widerhall in der Religionswissenschaft festzustellen. Cl. Schiwy $61 \mathrm{ff}$.

${ }^{38}$ Jaspers 1946, 277.

${ }^{39}$ Münzhuber 64:, Zeichen, die in sich keinen verstehbaren Gehalt bergen, sind von Menschen gemacht, um etwas ideal oder real 
keit ist es das Kennzeichen des Gegenstandes. Eine andere Eigenschaft besteht darin, daß man das Zeichen als solches von Fall zu Fall frei wählen und nach Belieben auch frei wechseln kann. Häufig gebraucht man in der Umgangssprache zur Bezeichnung der Staatsfahne oder des Staatswappens und ähnlichen ein symbolisches Wort. In Wirklichkeit sind dies Zeichen, die die oberste administrative Macht des Staates nach Belieben wechseln kann (zur Zeit wird in Schweden erwogen, das Staatswappen zu ändern). Zusammenfassend kann man mit Fawcett sagen: ,, The associations of the sign are with objective, empirical thinking, whereas the symbol (again, as we shall see) is associated with the emotional and existential levels of human operation." 40

Wiederholt ist $\mathrm{zu}$ betonen, daß, ,symbol is not created arbitrarily in the way that a sign can be. The symbol does, however, share with the sign the capacity to stand for something other than itself, but in a way which opens up possibilities which are closed to the sign. We have already noted that signs become really powerful, at least in a religious context, only when they are associated with a symbol. [...]

Symbols are not created, but born out of life. They do not come into being like signs as a result of the creative faculty of man's imagination." ${ }^{41}$

Einfacher gesagt, das Zeichen ist ein Hilfsmittel, ein Arbeitsrequisit, sowohl im täglichen Leben als auch in der wissenschaftlichen Arbeit, um die Orientierung und die Kommunikation innerhalb einer Gruppe zu erleichtern. Diese Kommunikation ist, dank der Konvention, rational, bestimmt und zielbewußt. Das Symbol hingegen gehört auf eine andere, psychische Ebene und wird hauptsächlich durch existentielle und emotionale Motive bestimmt. Mir scheint, daß Royce den strukturellen und funktionellen Unterschied zwischen Zeichen und Symbol in seinem Schema (siehe nächste Seite oben) sehr anschaulich dargestellt hat ${ }^{42}$.

Der hier aufgezeigte Unterschied der Termini ,Zeichen" und „Symbol" sollte in den Diskussionen beachtet werden, um diese zu vereinfachen und auf ein höheres Niveau der Sachlichkeit zu bringen.

5. Wir haben bereits beobachtet, daß im Zusammenhang mit der Frage

Seiendes zu vergegenwärtigen." Besondere Aufmerksamkeit haben der Unterscheidung von Zeichen und Symbol auch Thass-Thienemann, $17 \mathrm{ff}$, und Bertalanffy, $35 \mathrm{f}$, gewidmet. Cf. auch Fessard $291 \mathrm{ff}$.

40 Fawcett $14 \mathrm{f}$.

41 Ib. $26 \mathrm{f}$. Cf. auch Tillich 1964, 208. Die Forderung der Trennung von Zeichen und Symbol hat auch Wisse, $29 \mathrm{ff}$, anerkannt. In seiner Lösung schlägt er vor, das Symbol als eine untergeordnete Art der Gattung Zeichen mit einem Gradunterschied anzusehen (31), was bedeuten würde, daß das Symbol sui generis ein Zeichen wäre (unter direktem Einfluß von Looff 18). Dieser Lösung fehlt die Begründung, wie sie unsere Darstellung des Wesens des Symbols enthält. Eine weitere Motivierung für eine Unterscheidungslinie zwischen dem Symbol und Zeichen hat Zunini angegeben: ,, The line of demarcation between sign and symbol is that which divides the capacity of animals from that of man. Animals act in accordance with signs and configurations of signs, but they do not reach the level of symbol." (307)

42 Royce 17. 


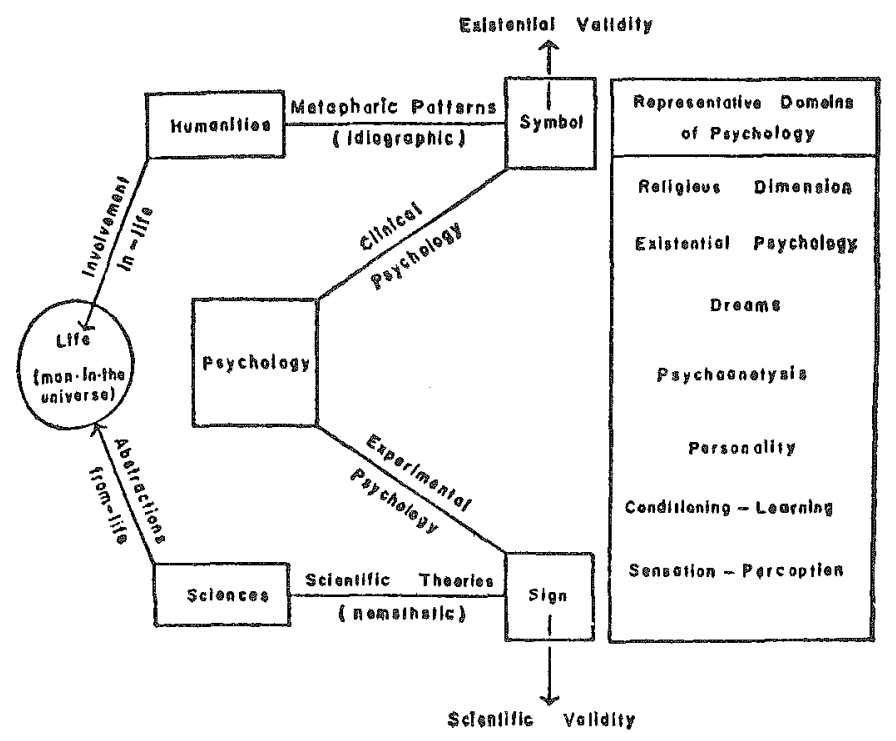

nach Zeichen und Symbolen auf die Kraft des Symbols hingewiesen wurde. Das eröffnet noch bessere Möglichkeiten, beide Erscheinungen zu unterscheiden. Es hilft auch, einen noch tieferen Einblick in das Wesen des Symbols zu gewinnen. Der Hinweis auf die Macht des Symbols ist ein sehr wichtiges Moment zum Verständnis des religiösen Symbols. Die Macht unterscheidet es seinem Wesen nach von den konventionellen, frei gewählten Zeichen, die den Erfordernissen einer Gruppe dienen. Der Unterschied ist nicht in der Notwendigkeit einer Kommunikation zu suchen. Diese besteht in allen Fällen. Im einen wie im anderen Fall hat man es mit einer kommunikativen Gruppe zu tun, deren Mitgliedern die von der Gruppe benutzten Symbole bzw. Zeichen bekannt sind. Es sind offensichtlich andere Umstände, die den religiösen Symbolen eine Kraft verleihen, die die Zeichen nicht besitzen. Welches sind diese Umstände? Die Macht des Symbols wurzelt im Wertbewußtsein. Wenn man diese Behauptung unter psychischem Aspekt betrachtet, kann man sagen, daß die Kräfte, die das Wertbewußtsein motivieren, größer sind als die Kraft der rational erkannten Wahrheit. Das kommt wahrscheinlich daher, daß im Wertbewußtsein emotionale, wenn nicht sogar biologische Motive mitsprechen ${ }^{43}$. Das

43 Eine einleuchtende psychologische Theorie der emotionellen Ausbildung des Gottessymbols gibt Ferroro 112f.: ,,Il y a donc, entre Dieu et son symbole, une confusion qui est très bien expliquée par la théorie de l'arrêt émotionnel. Dieu n'a jamais été vu par personne; on ne peut done en avoir qu'une image construite par l'imagination, sans l'aide des sensations, une image, pour ainsi dire, originelle: or, pour créer de toutes pièces, sans l'aide des sensations, une image assez vive, il faut un développement mental considérable et un effort assez grand. Aussi, dans la conscience du plus grand nombre des 
wird besonders deutlich in rational und emotional begründeten Konfliktfällen. Es bedeutet, daß das Wertbewußtsein den Menschen in der Tiefe seiner Existenz berührt ${ }^{44}$. Und hier kehren wir zurück zur existentiellen Bedeutung der Religion. Das religiöse Leben ist nicht beschränkt auf Aussagen durch formale begriffliche Konstruktionen. Es äußert sich immer in umfassenden Aktivitäten, ob auf individuellem oder sozialem Bereich des Lebens. Diese Aktivitäten sind wertbezogen und damit auch existenzbezogen ${ }^{45}$. Ich sehe es so, daß gerade diese Wertbezogenheit dem religiösen Symbol seine Kraft verleiht. Das bedeutet aber auch zugleich, daß das religiöse Symbol als begründete Ausdrucksform des Wertbewußtseins nach erkenntnistheoretischer Meinung nicht die Fähigkeit hat, die Wahrheit auszusagen $^{46}$. Wenn auch dem religiösen Symbol prinzipiell diese Eigenschaft fehlt, kann es dennoch vom psychischen Aspekt her die Qualität eines kognitiven Erlebnisses besitzen ${ }^{47}$. Im Symbolerlebnis spricht auch das Gefühl dafür mit, daß die symbolisierte Wirklichkeit die Wahrheit darstellt. Um der begrifflichen Klarheit willen müßte man in diesem Zusammenhang allerdings von Gewißheit sprechen.

hommes, au mot Dieu ne correspond-il qu'une image très vague et très peu précise. Il s'en suit que, lorsque le paysan voit une croix ou un autre symbole religieux réveillant en lui ces sentiments de crainte et de respect qui forment l'émotion religieuse, l'idée ou l'image de Dieu étant un état de conscience très peu clair et très peu précis, s'associe faiblement ou ne s'associe pas du tout à l'émotion. La sensation produite par la vue du symbole et les sentiments religieux sont seuls présents à l'esprit; ces sentiments ne peuvent donc avoir pour objet que le symbole, car la sensation du symbole est seule associée à l'émotion et la définit, tandis que l'idée et l'image de Dieu qui, dans l'acte psychique complet, devraient définir l'émotion, manquent. L'émotion s'arrête au symbole; et le symbole est adoré au lieu du Dieu qu'il devrait représenter." Cf. auch Ribot $393 \mathrm{f}$.

44 Auf die existentielle Bedeutung des Symbols ist wiederholt verwiesen worden, so bei Bertalanffy 40 ; Kahler 53 f. Fawcett spricht klar vom, ,existencial character of symbols": ,The existential character of symbols has already emerged quite clearly. They operate at the level of the subject and not that of objects. They are not part of our discursive discussions of that which lies outside the self, of our intellectual manipulation of reality as made up of series of objects. They are concerned with man's subjectivity and with the subjectivity of the cosmos itself. They work intuitively and directly out of man's experience of himself as self to grasp that subjectivity which lies in the whole realm of being" (33).

Auf die existenzielle Bindung hat auch Meslin richtig hingewiesen: ,Parce qu'il vise, en effet, à exprimer une réalité supérieure à laquelle il relie l'homme, le symbole religieux engage, dans une certaine mesure, l'existence même de l'homme qui le vit" (624).

45 Leese, 1948, 397, erwähnt in diesem $\mathrm{Zu}$ sammenhang die ,religiöse Wertigkeit, d.h. die erlebbare Werthöhe". Er sieht in ihr , das gesuchte Kriterium für die Wahrheit des religiösen Symbols" (ib. 405).

46 Sehr bestimmt hat sich darüber Jaspers, 1956, 151, geäußert: , Die Wahrheit der Chiffre aber kann ich nicht geradezu verifizieren, denn sie ist als ausgesprochene in ihrer $\mathrm{Ob}$ jektivität ein Spiel, das keinen Anspruch auf Geltung macht und daher auch keiner Rechtfertigung bedarf. Für mich selbst ist sie kein bloßes Spiel."

${ }^{47}$ Hier ist an den weitgefaßten Begriff der Rationalität bei Langer, Susanne, 99, zu erinnern: ,Rationality is the essence of mind, and symbolic transformation its elementary process. It is a fundamental error, therefore, to recognize it only in the phenomenon of systematic, explicit reasoning. That is a mature and precarious product.

Rationality, however, is embodied in every mental act, not only when the mind is 'at its fullest stretch and compass'." 
5.1. Die Richtigkeit der hier geäußerten theoretischen Gedankengänge kann überprüft werden, indem man sich in das sogenannte ,Werden und Vergehen" von Symbolen vertieft. In der Religionsgeschichte hat man es vielfach mit der ,Wiederbelebung" vergangener Symbole zu tun, d.h. damit, sie richtig zu begreifen. Im Laufe der Zeit haben einst aktuelle (, ,lebendige") Symbole ihre eigentliche Bedeutung verloren und sind nicht mehr verständlich. Mit anderen Worten, sie besitzen in der gegenwärtigen Situation bereits keine religiöse Funktion mehr. Die Religionsgeschichte kann ihnen ihre früheren Funktionen nicht mehr wiedergeben, d. h. sie kann sie nicht mehr ,wiederbeleben“. Um bei dieser Metapher zu bleiben: man kann sagen, daß die religionswissenschaftliche Forschung ein Spaziergang auf dem Friedhof der toten religiösen Symbole is $\mathrm{t}^{48}$. Das bezieht sich im selben Maß auch auf die Geschichte der christlichen Kirche. Aber weshalb vergehen Symbole? Die Antwort ist nicht schwer. In dem Augenblick, wo das Symbol nicht mehr dem Inhalt des religiösen Erlebnisses adäquat ist, stirbt es ab. Es hat keine Bedeutung mehr, es besitzt keinen aktuellen $\mathrm{Wert}^{49}$. Ein Symbol ohne Wert für das religiöse Leben ist mehr kein Symbol $^{50}$. Man weiß, daß der derzeitige Erzbischof der Kirche in Schweden Abbild und Kult des Gottes Thor kennt, der früher im alten Uppsala verehrt wurde, und eine Sammlung rationaler Erkenntnisse darüber besitzt. Er weiß, wer und was Thor ist und zu welchem religiösen Bereich er gehört. Aber das Abbild (Symbol) Thors und dessen Kult sind nicht Symbole seines religiösen Lebens. Mit anderen Worten, der Wert des religiösen Symbols von Thor ist verschwunden, denn das Oberhaupt einer christlichen Kirche des 20. Jahrhunderts hat sich in persönlicher Erfahrung eine eigene

48 Ähnlich hat sich Foss, 58, geäußert: ,Every langauge is full of 'dead metaphors'." Ebenso auch Bērzings 323. Ferroro, 186, spricht vom , Atavisme et pathologie du symbole".

${ }^{49}$ Ganz richtig verbindet Leese, 1948, 404, den Prozeß $\beta$ des Absterbens von Symbolen mit dem Wertbewußtsein: ,,Werden und Vergehen, Kampf und Verdrängung religiöser Symbole beruhen auf der jeweils labilen Vorzugsordnung religiös fundierter Werte, die immer mitgemeint sind [...]." Ebenso auch Davids, Rhys 154. Jaspers findet für das Absterben eine andere Erklärung. Die Symbole seien den dynamischen Prozessen des Lebens unterworfen. Sobald sie aus dem $\mathrm{Zu}$ stand, den er als ,die Schwebe" bezeichnet, zu einer statischen Erscheinung werden, sterben sie $a b$, d.h. sie werden zu zeichen: ,Aber Sprache, Wirklichkeit, Unendlichkeit eignen den Symbolen nur so lange, als sie in der Schwebe ihrer Erscheinung bleiben.
Sobald sie zu festen Bildern, fixierten Zeichen und damit zu Dingen in der Welt werden, geraten wir mit ihnen wieder an einen Strand, an den Strand falscher Leibhaftigkeiten, bloßer Bilder. Auch die Symbole, als verwandelte Objektivitäten, müssen in der Bewegung einer Ungreifbarkeit bleiben. Ihr Schwebesein macht allein ihre Unendlichkeit aus. Ihre Gestaltwerdung bringt sie auch auf den Weg, auf dem sie kollabieren zu festen Gebilden, die wie endliche Erkenntnisse genommen werden, aber nunmehr kein wirklicher Halt, sondern nur noch - am Realen gemessen - täuschende Illusionen sind." Cf. auch Meslin 624.

${ }^{50}$ Diesen Prozeß des Absterbens der Symbole spiegelt auch die sogenannte ,,Gott-isttot"-Theologie wider. Sie will sagen, daß das Wort ,Gott" keinen aktuellen religiösen Wert hat. Cf. zu dieser Frage die Schriften von Altizer $95 \mathrm{ff}$; Hamilton $23 \mathrm{ff}$; auch Christianity $315 \mathrm{ff}$; Vahanian 1961, $12 \mathrm{ff}$. 
religiöse Überzeugung gebildet und damit auch eine andere Welt der Symbole ${ }^{51}$.

Das ,Absterben“ religiöser Symbole ist eine Bestätigung des oben Gesagten, daß der religiöse Wert eines Symbols und seine Funktionen in einem Wertbewußtsein wurzeln ${ }^{52}$. Dieser Standpunkt läßt sich nur aufrecht erhalten, wenn man innerhalb der Grenzen des empirischen historischen Materials bleibt. Dagegen ist er hinfällig, wenn man die Auffassung einer ontologisch bestimmten bipolaren Struktur des religiösen Symbols vertreten will ${ }^{53}$. Natürlich kann man hier fragen, wodurch die Veränderung des Wertbewußtseins hervorgerufen und bestimmt wird. Diese Frage läßt sich nicht eindeutig beantworten, denn dann müßte man zugleich auch sagen können,

51 Cf. Fawcett 86 ff.: ,,Each symbolic expression in religion has enclosed within it an aspect of man's experience of the personal dimension. [...]

The power potentiality of a symbol depends upon the extent to which it corresponds to man's experience. That experience, however, constantly changes. The models which serve best in youth fall into the background in later life. The imagery which served ancient man so well may no longer speak to men in the twentieth century."

52 Eine ausgesprochen ablehnende Haltung gegenüber der Begründung des Symbols durch das Werbewußtsein hat Looff, 71, unter Kritik an der Auffassung Leeses, eingenommen: ,Wenn ich die Beziehung auf bestimmte Werte zum Maßstab der Wahrheit eines religiösen Symbols mache, setzt das immer ein durch eine bestimmte Werterziehung hindurchgegangenes Wertbewußtsein voraus, das dann mehr analog sich Symbole sucht und schafft, statt sich durch ein am Symbol aufleuchtendes Letztgemeintes erschüttern und in Frage stellen zu lassen. Dem einseitig religiös bestimmten Bewußtsein kann der größte religiöse Kitsch Ausdruck werden für das Unbedingt-Transzendente, wenn es darin seine geglaubte und bejahte Wertwelt wiederentdeckt. Bei einer Betonung der religiösen Wertigkeit des Symbols wird stets der mitgebrachte Glaube des Betrachters vorausgesetzt, der über die Ausdruckskraft des Symbols entscheidet."

Hierzu nur zwei Bemerkungen. Erstens hat Looff beim Gebrauch des Wortes ,Kitsch“" zur Bezeichnung des Wertbewußtseins, ausgehend von seiner religiösen Überzeugung, selbst ein Werturteil gefällt und damit bestätigt, daß religiöse Erlebnisse ein Wertbewußtsein darstellen. Zweitens scheint er nicht zu wissen, daß es sich um eine gewohnte Erscheinung in der Religions- geschichte handelt, wenn die Anhänger der einen Religion das ,Unbedingt-Transzendente" einer anderen Religion mit Worten belegen, die inhaltlich dem erwähnten ,Kitsch“ entsprechen. Daher berührt auch Looffs Kritik nicht das religiöse Wertbewußtsein, das seinen Ausdruck in Symbolen findet.

53 Als Tillich seinerzeit vom, Vergehen" der Symbole sprach, stieß er auf die schärfste Kritik gerade aus den konservativsten Kreisen in den USA, die diesem Gedanken nicht zustimmen konnten (cf. Modernists 5). Tilich mißverstehend, hat sich ihnen auch Ahlberg, $11 \mathrm{f}$, , mit seiner ÄuBerung , Gläubige ohne Glaube" angeschlossen. Die gleiche Reaktion ist auch von katholischer Seite erfolgt (Wisse 139). Wisse bekennt sich dazu wie folgt: , [...] ein Symbol, das zu irgendeiner Zeit wirklich existiert hat, [ist] unvergänglich, solange eben die Welt besteht" (139) und , das Symbol ist in sich [?], nach dem erstmaligen 'Geboren-Werden', unabhängig davon, ob es vom Menschen verstanden wird oder nicht" (139). Doch kann er nicht die Tatsache übersehen, daß Symbole verschwinden, „absterben“, Darauf antwortet er: ,Was als Vergehen oder Veränderung wahrgenommen wird, hat seinen Grund in Gegebenheiten auf seiten der Menschen" (210). Doch das Vergehen! Nun, der Mensch braucht und schafft sich Symbole, nicht aber Gott.

In ähnlicher Weise ist Eliade auf Irrwege geraten, wenn er meint, daß die Hierophanie, die sich einst in einem Symbol ausdrückte, nie wieder verschwindet: ,Ein Symbolismus ist unabhängig davon, ob er verstanden wird oder nicht, mehr, er bewahrt ihn sogar, wenn er ganz vergessen ist, was jene vorgeschichtlichen Symbole beweisen, deren Sinn über Tausende von Jahren vergessen war, um dann 'wiederentdeckt' zu werden" (510). 
wodurch überhaupt Veränderungen bestimmt werden. Eine Antwort auf diese Frage reicht ins Metaphysische. Anstelle einer Antwort muß man sich mit der Erkenntnis zufriedengeben, daß das Leben des Menschen in einer Welt verläuft, die ein dynamischer, ununterbrochener Prozeß von Veränderungen ist. Und in diesem dynamischen Prozeß entstehen und verschwinden auch die religiösen Symbole ${ }^{54}$.

\section{Literaturverzeichnis}

AUU Acta Universitatis Upsaliensis. Historia religionum. Uppsala.

EJ Eranos Jahrbuch. Zürich.

LL Le Langage. Neuchâtel.

$\mathrm{ZPhF}$ Zeitschrift für philosophische Forschung.

Ahlberg, A., 1966, Troende utan tro. Stockholm.

Altizer, T., 1966, Theology and the death of God. Theology.

Assagioli, R., 1969, Symbols of transpersonal experiences. The journal of transpersonal psychology.

Bertalanffy, L., 1965, On the definition of the symbol. Psychology.

Bērziņš, L., 1935, Latviešu tautas dziesmas [Lettische Volkslieder]. Latviešu literătūras vèsture 1. Rīgā.

Bevan, E., 1938, Symbolism and belief. London.

Bibliographie, $1968 \mathrm{f}$., Bibliographie zur Symbolik, Ikonographie und Mythologie $1 \mathrm{f}$. Hrsg. M. Lurker. Baden-Baden.

Biezais, H., 1961, Die Gottesgestalt der lettischen Volksreligion. AUU 1.

- 1972, Die himmlische Götterfamilie der alten Letten. AUU 5.

- 1976, Die Stellung der Religion in den soziokulturellen Strukturen. Dynamics and institution. Ed. H. Biezais. Scripta Instituti Donneriani Aboensis 9. Uppsala.

Cassirer, E., 1929, Philosophie der symbolischen Formen 3. Berlin.

Christianity, 1967, The new christianity. Ed. W. Miller. New York.

Davids, Rhys, 1934, Zur Geschichte des Rad-Symbols. EJ 2.

Eliade, M., s. a., Die Religionen und das Heilige. Salzburg.

Fawcett, T., 1970, The symbolic language of religion. Guildford.

Ferroro, G., 1895, Les lois psychologiques de symbolisme. Paris.

Fessard, G., 1966, Symbole symbolisant, signes, symbole symbolisé. $L L$ [1].

Fingesten, P., 1970, The eclipse of symbolism. Columbia.

Firth, R., 1973, Symbols, public and private. London.

Friedman, H., 1949, Wissenschaft und Symbol. München.

54 Über das Verschwinden bzw. Absterben von Symbolen im Christentum und $\mathrm{Ju}$ daismus sowie auch über das Entstehen neuer Symbole hat Lantis, Margaret 101, besonders 112, beachtenswerte Gedanken geäußert. Goodenough, $293 \mathrm{f}$., hat auf diese Weise ausgedruckt: , , [.. . ] that symbols have a way of dying, of apparently losing their power, and becoming merely ornaments. And they also have the power of coming to life again, as fresh associations and religious awakenings take one of the old symbols for their own. This happened when the Christians adopted the old, the universal symbol of the cross, a symbol which in pre-Christian ornament had degenerated to be only the four pointed rosette, one of many forms of the rosette. [...] For we must continue to face the problem of the 'merely decorative' as contrasted with the 'symbolic' use of forms in art; and when we contrast them in these terms, or in such terms as 'live' symbols versus 'dead' symbols, or 'active' symbols versus 'quiescent' or 'dormant' symbols in each case, we assume a theory of the nature of the contrast. Since I must have a terminology, I shall arbitrary, tentatively, and without prejudice, use the contrast 'live' and 'dead'." 
Giedion, S., 1966, Symbolic expression in prehistory and in the first high civilizations. Sign, image and symbol. Ed. G. Kepes. London.

Goodenough, E., 1952, The evaluation of symbols recurrent in time, as illustrated in Judaism. EJ 20 (1951).

Goodman, P., 1973, Speaking and language. London.

Hamilton, W., 1966, The death of God theology today. Theology.

Jaspers, K., 1946, Allgemeine Psychopathologie. Berlin.

- 1947, Von der Wahrheit. Philosophische Logik 1. München.

- 1956, Philosophie 3. Berlin.

Kahler, E., 1960, The nature of the symbol. Symbolism in religion and literature. Ed. R. May. New York.

Langer, Susanne, 1951, Philosophy in a new key. Cambridge.

Lantis, Margaret, 1950, The symbol of a new religion. Psychiatry 13.

Leese, K., 1934, Die Mutter als religiöses Symbol. Sammlung gemeinverständlicher Vorträge und Schriften aus dem Gebiet der Theologie und Religionsgeschichte 174. Tübingen.

- 1948, Die Religion des protestantischen Menschen. München.

Looff, H., 1955, Der Symbolbegriff in der neueren Religionsphilosophie und Theologie. Kant-Studien. Ergänzungshefte 69. Köln.

Mensching, G., 1930, Analyse des Symbolbegriffs. Christliche Welt 40.

- 1955, Religiöse Ursymbole der Menschheit. Studium generale 8.

- 1977, Topos und Typos. Untersuchungen zur allgemeinen Religionsgeschichte 8. Bonn.

Meslin, H., 1974, Pour une théorie du symbolisme religieux. Mélanges d'histoire des religions offert à Henri-Charles Puech. Paris.

Modernists, 1963, Modernists are not Christians. Lutheran news, Nov. 4.

Moody, R., 1956, On Jung's concept of the symbol. British journal of medical psychology 29.

Münzhuber, J., 1950, Sinnbild und Symbol. $Z P h F$.

Parsons, T., 1968, The structure of social action. London.

Psychology, 1965, Psychology and the symbol. Ed. J. Royce.

Ribot, T., 1915, La pensée symbolique. Revue philosophique 49.

Royce, J., 1965, Psychology at the crossroads between the sciences and the humanities. Psychology.

Saussure, F., 1916, Cours de linguistique générale. Paris.

Schiwy, G., 1973, Strukturalismus und Zeichensprache. Beck'sche Schwarze Reihe 96. München.

Segerstedt, T., 1947, Die Macht des Wortes. Internationale Bibliothek für Psychologie und Soziologie 3. Zürich.

Seiffert, A., 1957, Funktion und Hypertrophie des Sinnbildes. ZPhF. Beihefte 12.

Skorupski, J., 1976, Symbol and theory. Cambridge.

Symbolon, 1960 f., Symbolon 1 ff. Hrsg. J. Schwabe. Basel. N. F., 1 f. Hrsg. Reimbold. Köln.

Thass-Thienemann, T., 1968, Symbolic behavior. New York.

Theology, 1966, Radical theology and the death of God. Ed. T. Altizer, W. Hamilton. Indianapolis.

Tillich, P., 1928, Das religiöse Symbol. Blätter für deutsche Philosophie.

- 1964, Gesammelte Werke 5. Stuttgart.

- 1973, Symbol und Wirklichkeit. Kleine Vandenhoeck-Reihe 151. Göttingen.

Urban, W., 1939, Language and reality. London.

Vahanian, G., 1961, The death of God. New York.

Wisse, S., 1963, Das religiöse Symbol. Essen.

Zunini, G., 1969, Man and his religion. London. 